\title{
ON THE USE OF STATISTICAL PARALLAXES FOR THE PROPER MOTION REDUCTIONS
}

\author{
N.M. BRONNIKOVA, AND N.A. SHAKHT \\ Central Astronomical Observatory, USSR Academy of Sciences \\ Pulkovo \\ 196140 Leningrad \\ USSR
}

The Pulkovo program of determination of proper motions with respect to galaxies approaches completion. It consists of 157 fields from $+90^{\circ}$ to $-4^{\circ}$ of declination. The proper motions and reductions with use of galaxies were derived for all areas. The errors of reductions are equal to \pm 0 ":006. In addition the statistical reductions $R_{x}, R_{y}$ were computed for the control and for the zones of avoidance. The following formulae were used:

$$
R_{x}=(\bar{h} / \rho) P+Q ; \quad R_{y}=(\bar{h} / \rho) P^{\prime}+Q^{\prime} \text {, }
$$

where $(\overline{\mathrm{h}} / \mathrm{\rho})$ is the secular parallax according to Binnendijk [1]; P and $\mathrm{P}^{\prime}$ are parallactic factors; $Q$ and $Q^{\prime}$ are members dependent on the galactic rotation.

The nearby stars with large proper motions are able to give some systematic errors in reductions. The stars with proper motions greater than 0.05 arcsec per year were excluded in the Pulkovo catalogue treatment for the more uniform reference system.

At present the precise photographic magnitudes for all stars in 76 regions of the Pulkovo program were obtained. So it was possible to compute the values of secular parallaxes for reference stars with magnitudes from $12 \mathrm{~m} .5$ to $15^{\mathrm{m}} .5$. The parallaxes were computed with the formulae (1), where in the left parts the reductions obtained by use of galaxies were taken. The parameters for galactic rotation $Q, Q$ were adopted with standard values of galactic constants. The values of the parallaxes with their mean errors are given in Table 1.

Table 1.

The preliminary Pulkovo secular parallaxes ( in 0."0001).

\begin{tabular}{|c|c|c|c|c|c|c|c|c|c|}
\hline$m$ & $12 \mathrm{~m} .5$ & $13^{\mathrm{m}} \cdot 5$ & $14 \mathrm{~m} .5$ & 15.5 & & $12^{\mathrm{m}} \cdot 5$ & $13 \mathrm{~m} .5$ & $14 \mathrm{~m} .5$ & $15^{\mathrm{m}} \cdot 5$ \\
\hline $\begin{array}{l}\overline{\mathrm{h}} / \rho \\
|\overline{\mathrm{b}}|=28^{\circ}\end{array}$ & $\begin{array}{l}89 \\
\pm 26^{\circ}\end{array}$ & $\begin{array}{l}80 \\
\pm 26^{\circ}\end{array}$ & $\begin{array}{l}76 \\
\pm 19^{\circ}\end{array}$ & $\begin{array}{l}50 \\
\pm 20^{\circ}\end{array}$ & $|\bar{b}|=58^{\circ}$ & $\begin{array}{l}72 \\
\pm 25^{\circ}\end{array}$ & $\begin{array}{l}94 \\
\pm 20^{\circ}\end{array}$ & $\begin{array}{l}74 \\
\pm 20^{\circ}\end{array}$ & $\begin{array}{l}80 \\
\pm 16^{\circ}\end{array}$ \\
\hline$N$ & 13 & 10 & 23 & 22 & & 12 & 24 & 22 & 26 \\
\hline
\end{tabular}


Here $\mathrm{m}, \overline{\mathrm{b}}$ and $\mathrm{N}$ are the photographic magnitudes, average galactic latitude and numbers of areas. The total number of stars is about 9000 .

The stars with large proper motions have been not excluded from the data on which the parallaxes by Binnendijk were based. But there are the values of secular parallaxes by Deits [2] and Klemola and Vasilevskis [3] which were obtained on the basis of proper motions with similar cut-off. The comparison of Pulkovo parallaxes with [1], [2] and [3] indicate that the influence of large proper motions is more considerable in bright stars and in high latitudes.

We have deduced some analytic formulae for paralaxes of [2] and [3] in the form:

$$
\log p(m)=a+a_{1} m+a_{2} m^{2}
$$

where $p(m)$ is the mean parallax dependent on magnitude and calculated with velocity of Sun equal to $20 \mathrm{~km} / \mathrm{s}$. On the basis of formulae (2) some models of statistical reductions were made. Some differences between our galactic reductions and statistical reductions were considered.

In general these differences have reflected the distinction of parallaxes, but their behavior is complicated and the direct comparison of statistical reductions is difficult. A detailed discussion is planned in the future on the basis of all material. Nevertheless it seemed that the calculation of statistical reductions by means of the parallaxes from [2] and [3] based on the corresponding cut-off is more acceptable for our data.

\section{References}

[1] Binnendijk, L. (1943) “Mean parallaxes of faint stars, derived from a combination of the Pulkovo and Radcliffe Catalogue of proper motions", Bull. of the Astr. Inst. of the Netherlands, 10, \#362, 9-18.

[2] Deits, A.N. (1947) "Secular parallaxes of faint stars as deduced from Pulkovo Catalogue data on proper motions in Kapteyn Areas", Izv. GAO Pulkovo 17, \#138, 2-59. (in Russian).

[3] Klemola, A.R., Vasilevskis, S. (1971) "A study of solar motion and galactic rotation", Publ. Lick Obs. 22, Part 3, 1-13. 